\title{
Remarks on the fixed point problem of 2-metric spaces
}

\author{
Nguyen Van Dung, Nguyen Trung Hieu*, Nguyen Thi Thanh Ly and Vo Duc Thinh
}

"Correspondence:

ngtrunghieu@dthu.edu.vn;

ngtrunghieudhdt@gmail.com

Department of Mathematics, Dong

Thap University, Dong Thap, Dong

Thap, Vietnam

\begin{abstract}
In this paper, we prove a fixed point theorem on a 2-metric space and show that the main results in Lahiri et al. (Taiwan. J. Math. 15:337-352, 2011) and Singh et al. (J. Adv. Math. Stud. 5:71-76, 2012) may be obtained easily from the axioms of a 2-metric space. Examples are given to validate the results.
\end{abstract}

\section{Introduction and preliminaries}

There have been some generalizations of a metric space and its fixed point problem such as 2-metric spaces, $D$-metric spaces, $G$-metric spaces, cone metric spaces, complex-valued metric spaces. The notion of a 2-metric space was introduced by Gähler in [1]. Notice that a 2-metric is not a continuous function of its variables, whereas an ordinary metric is. This led Dhage to introduce the notion of a $D$-metric space in [2]. After that, in [3], Mustafa and Sims showed that most of topological properties of $D$-metric spaces were not correct. Then, in [4], they introduced the notion of a G-metric space and many fixed point theorems on G-metric spaces have been obtained. Unfortunately, in [5], Jleli and Samet showed that most of the obtained fixed point theorems on $G$-metric spaces can be deduced immediately from fixed point theorems on metric spaces or quasi-metric spaces. In [6], Huang and Zhang defined the notion of a cone metric space, which generalized a metric and a metric space, and proved some fixed point theorems for contractive maps on this space. After that, many authors extended some fixed point theorems on metric spaces to cone metric spaces. In [7], Feng and Mao introduced a metric on a cone metric space and then proved that a complete cone metric space is always a complete metric space. They verified that a contractive map on a cone metric space is a contractive map on a metric space, then fixed point theorems on a cone metric space are, essentially, fixed point theorems on a metric space. In [8], Azam, Fisher and Khan introduced the notion of a complex-valued metric space and some fixed point theorems on this space were stated. But in [9], Sastry, Naidu and Bekeshie showed that some fixed point theorems recently generalized to complex-valued metric spaces are consequences of their counter parts in the setting of metric spaces and hence are redundant.

Notice that in the above generalizations, only a 2 -metric space is not topologically equivalent to an ordinary metric. Then there was no easy relationship between results obtained in 2-metric spaces and metric spaces. In particular, the fixed point theorems on 2-metric spaces and metric spaces may be unrelated easily. For the fixed point theorems on 2-metric spaces, the readers may refer to [10-19].

o 2013 Dung et al:; licensee Springer. This is an Open Access article distributed under the terms of the Creative Commons Attribution License (http://creativecommons.org/licenses/by/2.0), which permits unrestricted use, distribution, and reproduction in any medium, provided the original work is properly cited. 
In this paper, we prove a fixed point theorem on a 2-metric space and show that the main results in [17] and [20] may be obtained easily from the axioms of a 2-metric space. Examples are given to validate the results.

Now we recall some notions and lemmas which will be useful in what follows.

Definition 1.1 ([1]) Let $X$ be a non-empty set and let $d: X \times X \times X \rightarrow \mathbb{R}$ be a map satisfying the following conditions:

1. For every pair of distinct points $a, b \in X$, there exists a point $c \in X$ such that $d(a, b, c) \neq 0$.

2. $d(a, b, c)=0$ only if at least two of three points are the same.

3. The symmetry: $d(a, b, c)=d(a, c, b)=d(b, c, a)=d(b, a, c)=d(c, a, b)=d(c, b, a)$ for all $a, b, c \in X$.

4. The rectangle inequality: $d(a, b, c) \leq d(a, b, d)+d(b, c, d)+d(c, a, d)$ for all $a, b, c, d \in X$.

Then $d$ is called a 2-metric on $X$ and $(X, d)$ is called a 2-metric space which will be sometimes denoted by $X$ if there is no confusion. Every member $x \in X$ is called a point in $X$.

\section{Remark 1.2}

1. Every 2-metric is non-negative.

2. We may assume that every 2-metric space contains at least three distinct points.

\section{Main results}

Theorem 2.1 Let $(X, d)$ be a 2-metric space and let $T, F: X \rightarrow X$ be two maps. If $d(T x, F y, x)=d(T x, F y, y)=0$ for all $x, y \in X$, then $T x$ is a fixed point of $T$ and $F y$ is a fixed point of $F$ for all $x, y \in X$.

Proof For all $x, y \in X$, we have

$$
d(T x, x, y) \leq d(T x, x, F y)+d(x, y, F y)+d(y, T x, F y)=d(F y, y, x) .
$$

By interchanging the roles of $x$ and $y, T$ and $F$, we get $d(F y, y, x) \leq d(T x, x, y)$. So,

$$
d(T x, x, y)=d(F y, y, x)
$$

for all $x, y \in X$. Then if $T x$ plays the role of $x$ in (2.1), we have $d\left(T^{2} x, T x, y\right)=d(F y, y, T x)=0$ for all $x, y \in X$. Hence $T^{2} x=T x$ for all $x \in X$. This proves that $T x$ is a fixed point of $T$ for all $x \in X$. Similarly, $F y$ is a fixed point of $F$ for all $y \in X$.

Corollary 2.2 ([21], Lemma 4.1) Let $(X, d)$ be a 2-metric space and let $T: X \longrightarrow X$ be a map. If $d(T x, T y, x)=0$ for all $x, y \in X$, then $T x$ is a fixed point of $T$ for all $x \in X$.

The following examples show that Theorem 2.1 is a proper generalization of Corollary 2.2.

Example 2.3 Let $X=\{1,2,3\}$ and $d(x, y, z)=\min \{|x-y|,|y-z|,|z-x|\}$ for all $x, y, z \in X$. Then $(X, d)$ is a 2-metric space. Let $T, F: X \longrightarrow X$ be two maps defined by $T 1=1, T 2=$ $T 3=3$ and $F 1=F 3=3, F 2=2$. We have $d(T 2, T 1,2)=d(3,1,2) \neq 0$ and $d(F 1, F 2,1)=$ 
Table 1 Calculations for maps in Example 2.3

\begin{tabular}{llll}
\hline $\boldsymbol{x}$ & $\boldsymbol{y}$ & $\boldsymbol{d}(\boldsymbol{T} \boldsymbol{x}, \boldsymbol{F y}, \boldsymbol{x})$ & $\boldsymbol{d}(\boldsymbol{T x}, \boldsymbol{F y}, \boldsymbol{y})$ \\
\hline 1 & 1 & $d(T 1, F 1,1)=d(1,3,1)=0$ & $d(T 1, F 1,1)=d(1,3,1)=0$ \\
1 & 2 & $d(T 1, F 2,1)=d(1,2,1)=0$ & $d(T 1, F 2,2)=d(1,2,2)=0$ \\
1 & 3 & $d(T 1, F 3,1)=d(1,3,1)=0$ & $d(T 1, F 3,3)=d(1,3,3)=0$ \\
2 & 1 & $d(T 2, F 1,2)=d(3,3,2)=0$ & $d(T 2, F 1,1)=d(3,3,1)=0$ \\
2 & 2 & $d(T 2, F 2,2)=d(3,2,2)=0$ & $d(T 2, F 2,2)=d(3,2,2)=0$ \\
2 & 3 & $d(T 2, F 3,2)=d(3,3,2)=0$ & $d(T 2, F 3,3)=d(3,3,3)=0$ \\
3 & 1 & $d(T 3, F 1,3)=d(3,3,3)=0$ & $d(T 3, F 1,1)=d(3,3,1)=0$ \\
3 & 2 & $d(T 3, F 2,3)=d(3,2,3)=0$ & $d(T 3, F 2,2)=d(3,2,2)=0$ \\
3 & 3 & $d(T 3, F 3,3)=d(3,3,3)=0$ & $d(T 3, F 3,3)=d(3,3,3)=0$ \\
\hline
\end{tabular}

$d(3,2,1) \neq 0$. This proves that Corollary 2.2 is neither applicable to $T$ nor $F$. On the other hand, Theorem 2.1 is applicable to $T$ and $F$ since $d(T x, F y, x)=d(T x, F y, y)=0$ for all $x, y \in$ $X$ as in the Table 1 .

Definition 2.4 ([17], Definition 12) Let $(X, d)$ be a 2-metric space and let $T: X \longrightarrow X$ be a map. $T$ is said to be contractive if $d(T x, T y, a)<d(x, y, a)$ for all $x \neq y \neq a \in X$, and $d(T x, T y, a)=0$ if any two of $x, y, a$ are equal.

Corollary 2.5 Let $(X, d)$ be a 2-metric space and let $T: X \rightarrow X$ be a contractive map. Then $T$ is a constant map, i.e., there exists $x_{0} \in X$ such that $T x=x_{0}$ for all $x \in X$. In particular, $T$ has a unique fixed point $x_{0}$ and the sequence $\left\{T^{n} x\right\}$ converges to $x_{0}$ for all $x \in X$.

Proof Since $d(T x, T y, x)=0$ for all $x \in X$, it follows from Corollary 2.2 that $T x$ is a fixed point of $T$ for all $x \in X$.

If $T x \neq T y$ for some $x, y \in X$, then there exists $a \in X$ such that $d(T x, T y, a) \neq 0$. Thus, $T x \neq T y \neq a$. Notice that $T^{2} x=T x$ and $T$ is a contractive map, so we have

$$
d(T x, T y, a)=d\left(T^{2} x, T^{2} y, a\right)<d(T x, T y, a) .
$$

It is a contradiction. Therefore $T x=T y$ for all $x, y \in X$, i.e., $T$ is a constant map. Let $T x=x_{0}$ for all $x \in X$. Then $x_{0}$ is the unique fixed point of $T$ and the sequence $\left\{T^{n} x\right\}$ converges to $x_{0}$ for all $x \in X$.

The following example shows that the contraction of $T$ in Corollary 2.5 is essential.

Example 2.6 Let $(X, d)$ be a 2-metric space and let $T: X \longrightarrow X$ be the identical map where $X$ has at least three points. Then $T$ is a non-contractive map with more than one fixed point.

In [17], Lahiri, Das and Dey established Cantor's intersection theorem and Baire category theorem in 2-metric spaces, and some fixed point theorems in 2-metric spaces have been proved sophisticatedly. By using the assumption of a contractive map, we show that the main results in [17] are direct consequences of Corollary 2.5. Moreover, the assumption of a contractive map is essential by Example 2.6.

Corollary 2.7 ([17], Theorem 7) Let $(X, d)$ be a complete bounded 2-metric space and let $T: X \longrightarrow X$ be a map such that $d(T x, T y, a) \leq \alpha \cdot d(x, y, a)$ for some $0<\alpha<1$ and all 
$x \neq y \neq a \in X$, and $d(T x, T y, a)=0$ if any two of $x, y, a \in X$ are equal. Then $T$ has a unique fixed point in $X$.

Corollary 2.8 ([17], Theorem 8$)$ Let $(X, d)$ be a bounded 2-metric space and let $T: X-$ $\rightarrow X$ be a map such that $d(T x, T y, a) \leq \alpha \cdot d(x, y, a)$ for some $0<\alpha<1$ and all $x, y, a \in X$. Let there be a point $x \in X$ such that the sequence of iterates $\left\{T^{n} x\right\}$ contains a subsequence $\left\{T^{n_{i}} x\right\}$ that converges to $x_{0} \in X$. Then $x_{0}$ is a unique fixed point of $T$.

Corollary 2.9 ([17], Theorem 9) Let $(X, d)$ be an uncounTable 2-metric space and let $T$ : $X \rightarrow X$ be a contractive map. If there exists a point $x \in X$ such that the sequence of iterates $\left\{T^{n} x\right\}$ contains a subsequence $\left\{T^{n_{i}} x\right\}$ converging to $x_{0} \in X$, then $x_{0}$ is the unique fixed point of $T$.

Recently, Singh, Mishra and Stofile have proved the following result.

Theorem 2.10 ([20], Theorem 2.1) Let $(X, d)$ be a complete 2-metric space and $T: X \longrightarrow$ $X$. Define a non-decreasing function $\theta$ from $[0,1)$ onto $\left(\frac{1}{2}, 1\right]$ by

$$
\theta(r)= \begin{cases}1 & \text { if } 0 \leq r \leq \frac{\sqrt{5}-1}{2} \\ \frac{1-r}{r^{2}} & \text { if } \frac{\sqrt{5}-1}{2} \leq r \leq \frac{1}{\sqrt{2}} \\ \frac{1}{1+r} & \text { if } \frac{1}{\sqrt{2}} \leq r<1\end{cases}
$$

Assume that there exists $r \in[0,1)$ such that

$$
\theta(r) \cdot d(x, T x, a) \leq d(x, y, a) \quad \text { implies } \quad d(T x, T y, a) \leq r \cdot d(x, y, a)
$$

for all $x, y, a \in X$. Then there exists a unique fixed point $z$ of $T$. Moreover, $\lim T^{n} x=z$ for any $x \in X$.

In the proof of the above theorem, Singh, Mishra and Stofile claimed that

$$
d\left(x_{n}, x_{n+1}, a\right) \leq d\left(x_{n}, z, a\right)+d\left(x_{n+1}, z, a\right)+d\left(x_{n}, x_{n+1}, x_{n}\right)
$$

in lines +4 and +5 , page 73 of [20]. In fact,

$$
d\left(x_{n}, x_{n+1}, a\right) \leq d\left(x_{n}, z, a\right)+d\left(x_{n+1}, z, a\right)+d\left(x_{n}, x_{n+1}, z\right) .
$$

The error inequality (2.3) was pointed out in [22].

Now, by choosing $a=x$ in (2.2), we have $\theta(r) \cdot d(x, T x, x)=0 \leq d(x, y, x)=0$. It implies that $d(T x, T y, x) \leq r \cdot d(x, y, x)=0$ for all $x, y \in X$. Then, by Corollary 2.2, $T$ has a fixed point. For the uniqueness, let $T$ have fixed points $x, y$. We have

$$
\theta(r) \cdot d(x, T x, a)=\theta(r) \cdot d(x, x, a)=0 \leq d(x, y, a) .
$$

It implies that $d(T x, T y, a)=d(x, y, a) \leq r \cdot d(x, y, a)$ for all $a \in X$. Then $d(x, y, a)=0$ for all $a \in X$, that is, $x=y$.

The following example shows that we cannot replace the assumption 'for all $x, y, a \in X$ ' in the contraction condition (2.2) by the assumption 'for all $x, y, a \in X$ and $a \neq x$ '. 
Table 2 Calculations for maps in Example 2.11

\begin{tabular}{lllll}
\hline $\boldsymbol{x}$ & $\boldsymbol{a}$ & $\boldsymbol{\theta}(\boldsymbol{r}) \cdot \boldsymbol{d}(\boldsymbol{x}, \boldsymbol{T x}, \boldsymbol{a}) \leq \boldsymbol{d}(\boldsymbol{x}, \boldsymbol{y}, \boldsymbol{a})$ & $\boldsymbol{y}$ & $\boldsymbol{d}(\mathbf{T x}, \mathbf{T y}, \boldsymbol{a}) \leq \boldsymbol{r} \cdot \boldsymbol{d}(\mathbf{x}, \boldsymbol{y}, \boldsymbol{a})$ \\
\hline 1 & 2 & $\theta(r) \cdot d(1, T 1,2)=0 \leq d(1, y, 2)$ & 1 & $d(T 1, T 1,2)=0 \leq r \cdot d(1,1,2)$ \\
& & & 2 & $d(T 1, T 2,2)=0 \leq r \cdot d(1,2,2)$ \\
& & & 3 & $d(T 1, T 3,2)=0 \leq r \cdot d(1,3,2)$ \\
1 & 3 & $\theta(r) \cdot d(1, T 1,3)=\theta(r) \cdot d(1,2,3) \leq d(1, y, 3)$ & 2 & $d(T 1, T 2,3)=0 \leq r \cdot d(1,2,3)$ \\
2 & 3 & $\theta(r) \cdot d(2, T 2,3)=0 \leq d(2, y, 3)$ & 1 & $d(T 2, T 1,3)=0 \leq r \cdot d(2,1,3)$ \\
& & 2 & $d(T 2, T 2,3)=0 \leq r \cdot d(2,2,3)$ \\
& & & 3 & $d(T 2, T 3,3)=0 \leq r \cdot d(2,3,3)$ \\
2 & 1 & $\theta(r) \cdot d(2, T 2,1)=\theta(r) \cdot d(2,3,1) \leq d(2, y, 1)$ & 3 & $d(T 2, T 3,1)=0 \leq r \cdot d(2,3,1)$ \\
3 & 1 & $\theta(r) \cdot d(3, T 3,1)=0 \leq d(1, y, 3)$ & 1 & $d(T 3, T 1,1)=0 \leq r \cdot d(3,1,1)$ \\
& & & 2 & $d(T 3, T 2,1)=0 \leq r \cdot d(3,2,1)$ \\
& & & 3 & $d(T 3, T 3,1)=0 \leq r \cdot d(3,3,1)$ \\
3 & 2 & $\theta(r) \cdot d(3, T 3,2)=\theta(r) \cdot d(3,1,2) \leq d(2, y, 3)$ & 1 & $d(T 3, T 1,2)=0 \leq r \cdot d(3,1,2)$
\end{tabular}

Example 2.11 Let $X=\{1,2,3\}$ and $d(x, y, z)=\min \{|x-y|,|y-z|,|z-x|\}$ for all $x, y, z \in X$. Then $(X, d)$ is a complete 2 -metric space. Let $T: X \rightarrow X$ be a map defined by $T 1=2$, $T 2=3, T 3=1$. We see that $T$ has no fixed point. But, for all $x, y, a \in X$ and $a \neq x$, the contraction condition (2.2) holds as in the Table 2.

Competing interests

The authors declare that they have no competing interests.

Authors' contributions

All the authors contributed equally. All authors read and approved the final manuscript.

\section{Acknowledgements}

The authors would like to thank the referees for their valuable comments.

Received: 20 December 2012 Accepted: 4 June 2013 Published: 26 June 2013

\section{References}

1. Gähler, VS: 2-metrische Räume und ihre topologische struktur. Math. Nachr. 26, 115-118 (1963/64)

2. Dhage, BC: A study of some fixed point theorems. Ph.D. thesis, Marathwada, Aurangabad, India (1984)

3. Mustafa, Z, Sims, B: Some remarks concerning D-metric spaces. In: Proceedings of the International Conferences on Fixed Point Theory and Applications, Valencia, Spain, pp. 189-198 (2003)

4. Mustafa, Z, Sims, B: A new approach to generalized metric spaces. J. Nonlinear Convex Anal. 7(2), $289-297$ (2006)

5. Jleli, M, Samet, B: Remarks on G-metric spaces and fixed point theorems. Fixed Point Theory Appl. 2012, Article ID 201 (2012)

6. Huang, LG, Zhang, X: Cone metric spaces and fixed point theorems of contractive mappings. J. Math. Anal. Appl. 332, 1468-1476 (2007)

7. Feng, Y, Mao, W: The equivalence of cone metric spaces and metric spaces. Fixed Point Theory 11(2), 259-264 (2010)

8. Azam, A, Fisher, B, Khan, M: Common fixed point theorems in complex valued metric spaces. Numer. Funct. Anal. Optim. 32(3), 243-253 (2011)

9. Sastry, KPR, Naidu, GA, Bekeshie, T: Metrizability of complex valued metric spaces and some remarks on fixed point theorems in complex valued metric spaces. Int. J. Pure Appl. Math. 3(7), 2686-2690 (2012)

10. Aliouche, A, Simpson, C: Fixed points and lines in 2-metric spaces. Adv. Math. 229, 668-690 (2012)

11. Deshpande, B, Chouhan, S: Common fixed point theorems for hybrid pairs of mappings with some weaker conditions in 2-metric spaces. Fasc. Math. 46, 37-55 (2011)

12. Freese, RW, Cho, YJ, Kim, SS: Strictly 2-convex linear 2-normed spaces. J. Korean Math. Soc. 29(2), 391-400 (1992)

13. Gähler, VS: Lineare 2-normierte Räume. Math. Nachr. 28, 1-43 (1965)

14. Gähler, VS: Über die uniformisierbarkeit 2-metrischer Räume. Math. Nachr. 28, 235-244 (1965)

15. Iseki, K: Fixed point theorems in 2-metric spaces. Math. Sem. Notes Kobe Univ. 3, 133-136 (1975)

16. Iseki, K: Mathematics on 2-normed spaces. Bull. Korean Math. Soc. 13(2), 127-135 (1976)

17. Lahiri, BK, Das, P, Dey, LK: Cantor's theorem in 2-metric spaces and its applications to fixed point problems. Taiwan. J. Math. 15, 337-352 (2011)

18. Lai, SN, Singh, AK: An analogue of Banach's contraction principle of 2-metric spaces. Bull. Aust. Math. Soc. 18, 137-143 (1978)

19. Vats, RK, Kumar, S Sihag, V: Fixed point theorems in complete G-metric space. Fasc. Math. 47, 127-139 (2011)

20. Singh, SL, Mishra, SN, Stofile, S: Suzuki contraction theorem on a 2-metric space. J. Adv. Math. Stud. 5, 71-76 (2012)

21. An, TV, Dung, NV, Hieu, NT: Further results on 2-metric spaces. J. Sci. Vinh Univ. 41(3), 1-10 (2012)

22. Singh, SL, Mishra, SN, Stofile, S: Erratum to 'Suzuki contraction theorem on a 2-metric space'. J. Adv. Math. Stud. 5(2), $138(2012)$ 
doi:10.1186/1687-1812-2013-167

Cite this article as: Dung et al.: Remarks on the fixed point problem of 2-metric spaces. Fixed Point Theory and Applications 2013 2013:167.

Submit your manuscript to a SpringerOpen ${ }^{\ominus}$ journal and benefit from:

- Convenient online submission

- Rigorous peer review

- Immediate publication on acceptance

- Open access: articles freely available online

- High visibility within the field

- Retaining the copyright to your article

Submit your next manuscript at $\gg$ springeropen.com 